\title{
Supplementary Material: Fast Digital Lossy Compression for X-ray Ptychographic Data
}

The crossing between Fourier ring correlation (FRC) and 1/2 bit threshold is a widely used standard to determine the achieved resolution of the reconstructed image in ptychography. Here we derive the formula of $1 / 2$ bit threshold when the FRC is calculated using 2 full images with independent noise.

Given the required SNR, the corresponding Fourier correlation (which in the $2 \mathrm{D}$ case is a Fourier ring correlation or FRC rather than a Fourier shell correlation) can be calculated using Eq. 13 of [1], which we rewrite here as

$$
\operatorname{FRC}\left(r_{i}\right)=\frac{\operatorname{SNR}\left(r_{i}\right)+2 / \sqrt{n\left(r_{i}\right)} \sqrt{\operatorname{SNR}\left(r_{i}\right)}+1 / \sqrt{n\left(r_{i}\right)}}{\operatorname{SNR}\left(r_{i}\right)+2 / \sqrt{n\left(r_{i}\right)} \sqrt{\operatorname{SNR}\left(r_{i}\right)}+1}
$$

where $n\left(r_{i}\right)$ is the number of voxels in the Fourier shell, $r_{i}$. The required SNR of the 1/2-bit threshold can be calculated using

$$
\frac{1}{2} \text { bit }=\log _{2}(1+\mathrm{SNR})=\log _{2}(1+0.4142)
$$

from which we obtained the requirement of $\mathrm{SNR}=0.4142$ in the full image.

In the case of calculating the FRC between 2 half images, the required $\mathrm{SNR}$ is in each of the half images is also half $(0.4142 / 2=0.2071)$ because the total SNR is twice the SNR value of each of the half images. The $1 / 2$-bit threshold then can be calculated by plugging $\operatorname{SNR}\left(r_{i}\right)=0.2071$ into Eq. (1) to get Eq. 17 of [1], which we rewrite here as

$$
T_{1 / 2-\text { bit }}=\frac{0.2071+1.9102 / \sqrt{n_{r_{i}}}}{1.2071+1.9102 / \sqrt{n_{r_{i}}}}
$$


In our work, we calculated the FRC between 2 full images. Therefore, we plugged the value $\mathrm{SNR}=0.4142$ directly into Eq. (1), which gives

$$
T_{1 / 2-\text { bit }}=\frac{0.4142+2.2872 / \sqrt{n_{r_{i}}}}{1.4142+1.2872 / \sqrt{n_{r_{i}}}} .
$$

\section{References}

[1] Marin van Heel and Michael Schatz. Fourier shell correlation threshold criteria. Journal of Structural Biology, 151(3):250-262, September 2005. 